\title{
The Projective Group as a Topological Manifold
}

\author{
Jean-Francois Niglio \\ Department of Mathematics, Kingston University, London, UK \\ Email: jeanfrancois.niglio@gmail.com
}

How to cite this paper: Niglio, J.-F. (2018) The Projective Group as a Topological Manifold. Advances in Linear Algebra \& Matrix Theory, 8, 135-142.

https://doi.org/10.4236/alamt.2018.84012

Received: October 23, 2018

Accepted: December 3, 2018

Published: December 6, 2018

Copyright $\odot 2018$ by author and Scientific Research Publishing Inc. This work is licensed under the Creative Commons Attribution International License (CC BY 4.0).

http://creativecommons.org/licenses/by/4.0/

\begin{abstract}
In this article, we start by a review of the circle group $\mathbb{T}[1]$ and its topology induced [1] by the quotient metric, which we later use to define a topological structure on the unit circle $\left(\mathbb{S}^{1}, \tau_{\mathbb{S}^{1}}\right)$. Using points on $\mathbb{S}^{1}$ under the complex exponential map, we can construct orthogonal projection operators. We will show that under this construction, we arrive at a topological group, denoted $G_{P}([\theta])$ of projection matrices. Together with the induced topology, it will be demonstrated that $G_{P}([\theta])$ is Hausdorff and Second Countable forming a topological manifold. Moreover, I will use an example of a group action on $G_{P}([\theta])$ to generate subgroups of $G_{p}([\theta])$.
\end{abstract}

\section{Keywords}

Projection, Orthogonal Projections, Projective Operators, Projective Manifolds

\section{Introduction}

Orthogonal Projection is a very familiar topic in Linear Algebra [2]. With reference to [2], it is already known that if $V$ is a finite-dimensional vector space and $P$ is a projection on $W \subset V$, where $W$ is a subspace of $V$. Then $P$ is idempotent, that is $P^{2}=P$. $P$ is the identity operator on $W$, that is $\forall x \in W: P x=x$. We also know that $W$ is the range of $P$ and if $U$ is the kernel of $P$ then $V=W \oplus U, w \in W, u \in U$. It is easy to show that $w=P x, u=(I-P) x$. It is also a known fact that these operators are bounded i.e. $\|P x\| \leq\|x\|$. In this paper we will focus on projections in $\mathbb{R}^{2}$ and define a different construct for these operators. Starting in the next section with the circle group $\mathbb{T}$ [1] it is possible to endow the set of projective operators with a group and topological structure. 


\section{Notation Used in This Article}

1) $\left(\mathbb{S}^{1}, \tau_{\mathbb{S}^{1}}\right)$ The unit circle as a Topological Group.

2) $\mathbb{T}$ The circle group defined as $\mathbb{T}:=\mathbb{R} / 2 \pi \mathbb{Z}$.

3) $[\theta]$ is an element in the topology of $\mathbb{T}$.

4) $\tau_{\mathbb{R}}$ the usual topology on $\mathbb{R}$.

5) $\tau_{\mathbb{S}^{1}}$ the topology on the unit circle $\mathbb{S}^{1}$.

6) $d_{\mathbb{T}}\left(\left[\theta_{1}\right],\left[\theta_{2}\right]\right)$ is the Quotient Metric on $\mathbb{T}$.

7) $\mathcal{B}([\theta], \epsilon)$ opens balls in $\mathbb{T}$.

8) $\mathcal{B}(\theta, \epsilon)$ is open in $\mathbb{R}$.

9) $\left(\mathbb{T}, \cdot, \tau_{-} \mathbb{T}\right)$ the circle group as a topological group.

10) $\left(\mathbb{S}^{1}, \cdot, \tau_{\mathbb{S}^{1}}\right)$ the unit circle as topological group.

11) $\mathcal{O}_{\mathbb{S}^{1}}^{\alpha_{+}}$open set in $\mathbb{S}^{1}$ generated by counter-clockwise rotation.

12) $\mathcal{O}_{\mathbb{S}^{-}}^{\alpha_{-}}$open set in $\mathbb{S}^{1}$ generated by clockwise rotation.

13) $P_{[\theta]}$ A projection matrix at angle $[\theta]$.

14) $G_{p}([\theta])$ the topological projection manifold.

15) $\mathcal{P}_{\alpha}$ open sets in $G_{p}([\theta])$.

\section{A Brief Review of the Circle Group $\mathbb{T}$ and Its Topology}

\subsection{The Group Structure}

The topological group as in [1] is constructed by viewing the real line $\mathbb{R}$ as topological group where we identify $x=\theta$. The topology is the usual topology induced by the metric $d_{\mathbb{R}}\left(\theta_{1}, \theta_{2}\right):=\left|\theta_{1}-\theta_{2}\right|$. We then define the following equivalence relation

$$
\theta_{1} \sim \theta_{2} \Leftrightarrow \theta_{1}-\theta_{2}=2 \pi k, k \in \mathbb{Z}
$$

Without proof, we see that this is an equivalence relation. The circle group is then defined as

$$
\mathbb{T}:=\mathbb{R} / 2 \pi \mathbb{Z}
$$

That is,

$$
\mathbb{T}:=\{[\theta] \mid \theta \in \mathbb{R},[\theta]=\{\theta+2 \pi k, k \in \mathbb{Z}\}\}
$$

It is very clear that this forms a group under addition such that

$$
\left[\theta_{1}\right]+\left[\theta_{2}\right]:=\left[\theta_{1}+\theta_{2}\right]
$$

With the identity $\theta=0$

$$
\left[\theta_{1}\right]+[0]=\left[\theta_{1}+0\right]=\left[\theta_{1}\right]
$$

The additive inverse is simply given by $[\theta]^{-1}:=\left[-\theta_{1}\right]=-\left[\theta_{1}\right]$ such that

$$
[\theta]+[\theta]^{-1}=\left[\theta_{1}\right]+\left[-\theta_{1}\right]=\left[\theta_{1}-\theta_{1}\right]=[0]
$$

where $[0]:=\{2 \pi k, k \in \mathbb{Z}\}$.

It is clear that this is associative and hence $\mathbb{T}$ is a group.

\subsection{The Topological Structure on $\mathbb{T}[1][3]$}

First, we see that we have the projection mapping such that 


$$
\pi: \mathbb{R} \rightarrow \mathbb{T}
$$

Such that

$$
\pi(\theta)=[\theta]
$$

Since $\mathbb{R}$ is a topological space we can define the topology on $\mathbb{T}$ be declaring an open set $U \in \mathbb{T}$ to be open if and only if $\pi^{-1}(U)$ is open in $\mathbb{R}$.

Suppose we have some open $\mathcal{O} \in \mathbb{R}$ then we have $\pi(\mathcal{O})=\{[\theta] \mid \theta \in \mathcal{O}\}$ hence we define $U:=\{[\theta] \mid \theta \in \mathcal{O}\}$ to be open in $\mathbb{T}$. It is clear that $\pi^{-1}(U)$ is open in $\mathbb{R}$. Next, we show the closure w.r.t unions and intersections. From topology, it is well known that the union of open sets is open and the intersection of open sets is also open, therefore, we can see that

$$
\bigcup_{\alpha} U_{\alpha} \in \tau \Rightarrow \pi^{-1}\left(\bigcup_{\alpha} U_{\alpha}\right)=\bigcup_{\alpha} \pi^{-1}\left(U_{\alpha}\right) \in \tau_{\mathbb{R}}, \alpha \in I
$$

For the intersections we get

$$
\pi^{-1}\left(\bigcap_{\alpha} U_{\alpha}\right)=\bigcap_{\alpha} \pi^{-1}\left(U_{\alpha}\right), \alpha \in I
$$

Clearly, $\pi^{-1}\left(U_{\alpha}\right)$ is open in $\mathbb{R}$ since $\pi^{-1}\left(U_{\alpha}\right)=\mathcal{O}_{\alpha}$.

\subsection{The Quotient Metric on the Circle Group}

The quotient topology $\tau_{\mathbb{T}}$ is induced by the quotient metric defined as

$$
d_{\mathbb{T}}\left(\left[\theta_{1}\right],\left[\theta_{2}\right]\right):=\inf _{k \in \mathbb{Z}}\left\{\left|\theta_{1}-\theta_{2}+2 \pi k\right|\right\}
$$

We can define an open ball from this metric in the following way

$$
\mathcal{B}([\theta], \epsilon):=\left\{\left[\theta^{\prime}\right] \in \mathbb{T}: d_{-} \mathbb{T}\left([\theta],\left[\theta^{\prime}\right]\right)<\epsilon, \epsilon>0\right\}
$$

Let $[\theta] \in \mathbb{T}$ and $q_{k}$ be the $k^{\text {th }}$ representative of $[\theta]$, that is $q_{k}:=\theta+2 \pi k$ for some $k \in \mathbb{Z}$. Let $\left[\theta^{\prime}\right]$ be some other point in $\mathbb{T}$. Then we think of $d_{\mathbb{T}}:=\inf _{k \in \mathbb{Z}}\left\{d\left(q_{k}, \theta^{\prime}\right)\left|d\left(q_{k}, \theta^{\prime}\right)=\right| q_{k}-\theta^{\prime} \mid\right\}$. Hence, the open sets in $\mathbb{T}$ can be defined by using the definition of the open balls (above) and the canonical projection mapping $\pi$.

In order for the canonical map $\pi$ to make sense and in order to satisfy $\inf _{k \in \mathbb{Z}}\left\{d\left(q_{k}, \theta^{\prime}\right)\left|d\left(q_{k}, \theta^{\prime}\right)=\right| q_{k}-\theta^{\prime} \mid\right\}$ we construct the open balls as discussed above

$$
\mathcal{B}([\theta], \epsilon):=\left\{\left[\theta^{\prime}\right]\left|\theta^{\prime} \in \mathcal{O}, d\left(\theta, \theta^{\prime}\right)=\right| \theta-\theta^{\prime} \mid<\epsilon, \epsilon>0\right\}
$$

Note that

$$
\pi^{-1}(\mathcal{B}([\theta], \epsilon))=\mathcal{B}(\theta, \epsilon)
$$

Such that

$$
\mathcal{B}(\theta, \epsilon):=\left\{\theta^{\prime} \in \mathbb{R}\left|d\left(\theta, \theta^{\prime}\right)=\right| \theta-\theta^{\prime} \mid<\epsilon, \theta^{\prime} \in \mathcal{O}_{\alpha}, \epsilon>0\right\}
$$

This defines a topology on $\mathbb{T}$. Furthermore, even though $\mathcal{B}(\theta, \epsilon)$ is open and bounded in $\mathbb{R}$, any open set in $\mathbb{R}$ can be written as the countable union of open balls $\mathcal{B}(\theta, \epsilon)$. 
Also we note that this metric is a pseudo-metric. A pseudo-metric is metric is similar to usual metric spaces with the exception that it possible to have the following result

$$
d_{\mathbb{T}}\left([\theta],\left[\theta^{\prime}\right]\right)=0 \nRightarrow \theta=\theta^{\prime}
$$

In fact, this implies that $\theta^{\prime}=q_{k}=\theta+2 \pi k, k \in \mathbb{Z} \Rightarrow[\theta]=\left[q_{k}\right], \forall k \in \mathbb{Z}$.

Hence, $\mathbb{T}$ is a topological group, denoted $\left(\mathbb{T},+, \tau_{-} \mathbb{T}\right)$.

\section{The Mapping $I: \mathbb{T} \rightarrow \mathbb{S}^{1}$}

We now consider the unit circle as the set

$$
\mathbb{S}^{1}:=\left\{x \in \mathbb{R}^{2}: x^{2}+y^{2}=1\right\}
$$

Clearly, $\mathbb{S}^{1}$ can e endowed with the subspace topology $\tau_{\mathbb{S}^{1}}$ generated by the metric $d: \mathbb{S}^{1} \times \mathbb{S}^{1} \rightarrow[0, \infty)$ such that we define the metric to be $d\left(\mathrm{e}^{i \theta}, \mathrm{e}^{i \theta^{\prime}}\right)$ i.e. the shortest arc length between the points $\left(\mathrm{e}^{i \theta}, \mathrm{e}^{i \theta^{\prime}}\right)$.

Clearly, this defines a topology on $\mathbb{S}^{1}$. Equipped with this topology we can say that $\left(\mathbb{S}^{1}, \cdot, \tau_{\mathbb{S}^{1}}\right)$ is a topological group. The mapping

$$
I:\left(\mathbb{T},+, \tau_{\mathbb{T}}\right) \rightarrow\left(\mathbb{S}, \cdot, \tau_{\mathbb{S}^{1}}\right)
$$

such that

$$
I([\theta]):=\mathrm{e}^{i[\theta]},[\theta] \in \mathbb{T}
$$

This, clearly defines an isomorphism. The group operation on the circle is given by multiplication as follows

$$
\mathrm{e}^{i \theta} \mathrm{e}^{i \theta^{\prime}}=\mathrm{e}^{i\left(\theta+\theta^{\prime}\right)}=I\left([\theta]+\left[\theta^{\prime}\right]\right)
$$

Furthermore

$$
\begin{aligned}
& \mathrm{e}^{i \theta} \mathrm{e}^{i \theta^{\prime}}=(\cos \theta+i \sin \theta)\left(\cos \theta^{\prime}+i \sin \theta^{\prime}\right) \\
& =\cos \theta \cos \theta^{\prime}+i \cos \theta \sin \theta^{\prime}+i \sin \theta \cos \theta^{\prime}-\sin \theta \sin \theta^{\prime} \\
& =\cos \left(\theta+\theta^{\prime}\right)+i \sin \left(\theta+\theta^{\prime}\right)=I\left([\theta]+\left[\theta^{\prime}\right]\right)
\end{aligned}
$$

With

$$
d_{\mathbb{T}}\left([\theta],\left[\theta^{\prime}\right]\right)=d\left(\mathrm{e}^{i \theta}, \mathrm{e}^{i \theta^{\prime}}\right)
$$

It is clear that open balls on $\mathbb{T}$ are mapped into open arcs on $\mathbb{S}^{1}$.

\section{Defining Projections on $\left(\mathbb{S}^{1}, \cdot, \tau_{\mathbb{S}^{1}}\right)$}

We now focus on the topology generated by open arcs. We can write

$$
\begin{gathered}
\mathrm{e}^{i \theta}=\cos \theta+i \sin \theta=(\cos \theta, \sin \theta)^{\mathrm{T}} \in \mathbb{S}^{1} \\
\mathrm{e}^{-i \theta}=\cos \theta-i \sin \theta=(\cos \theta,-\sin \theta)^{\mathrm{T}}, \in \mathbb{S}^{1}
\end{gathered}
$$


We define the following mapping

$$
\Phi\left(\mathrm{e}^{i[\theta]}\right):=\left\{\begin{array}{lc}
\mathrm{e}^{i[\theta]} \otimes\left(\mathrm{e}^{i[\theta]}\right)^{\mathrm{T}}, & \forall \theta \in \mathcal{O}_{\mathbb{S}^{+}}^{\alpha_{+}} \\
P_{e}, & \theta=0 \\
\mathrm{e}^{i[-\theta]} \otimes\left(\mathrm{e}^{i[-\theta]}\right)^{\mathrm{T}}, & \forall \theta \in \mathcal{O}_{\mathbb{S}^{1}}^{\alpha_{-}}
\end{array}\right.
$$

Given that each open set in the topology satisfies the metric $d\left(\mathrm{e}^{i \theta}, \mathrm{e}^{i \theta^{\prime}}\right)<\pi$ implies that $\Phi$ is bijective and hence has an inverse.

Moreover, we have

$$
\Phi\left(\mathrm{e}^{i[\theta]} \mathrm{e}^{i\left[\theta^{\prime}\right]}\right)=\Phi\left(\mathrm{e}^{i\left[\theta+\theta^{\prime}\right]}\right)=\mathrm{e}^{i\left[\theta+\theta^{\prime}\right]} \otimes\left(\mathrm{e}^{i\left[\theta+\theta^{\prime}\right]}\right)^{\mathrm{T}}
$$

The group operation of the projector is defined as follows

$$
\varphi\left(P_{[\theta]}, P_{\left[\theta^{\prime}\right]}\right)=P_{\left[\theta+\theta^{\prime}\right]}
$$

where $P_{[\theta]}=\mathrm{e}^{\mathrm{i}[\theta]} \otimes\left(\mathrm{e}^{\mathrm{i}\left[\theta^{\prime}\right]}\right)^{\mathrm{T}}$ and $P_{\left[\theta+\theta^{\prime}\right]}=\mathrm{e}^{\left[\theta+\theta^{\prime}\right]} \otimes\left(\mathrm{e}^{\left[\theta+\theta^{\prime}\right]}\right)^{\mathrm{T}}$ respectively.

Clearly, it can be easily verified that this defines an abelian group as per definition from [4] [5]. Also, this is consistent with the group operation on $\mathbb{S}^{1}$ and shows that $\Phi$ is a group homomorphism.

\section{Image and Kernel}

Let $u \in \mathbb{R}^{2}$. The vector can be written as $u=\left(\|u\| \cos \theta_{u},\|u\| \sin \theta_{u}\right) . \quad \theta_{u}$ denotes the angle between vector $u$ and the $\mathrm{x}$-axis. Then we have

$$
\begin{aligned}
& \mathrm{e}^{i[\theta]} \otimes\left(\mathrm{e}^{i[\theta]}\right)^{\mathrm{T}} u=\left[\begin{array}{cc}
\cos ^{2} \theta & \cos \theta \sin \theta \\
\sin \theta \cos \theta & \sin ^{2} \theta
\end{array}\right]\left[\begin{array}{l}
\|u\| \cos \theta_{u} \\
\|u\| \sin \theta_{u}
\end{array}\right] \\
& =\|u\|\left[\begin{array}{c}
\cos ^{2} \theta \cos \theta_{u}+\cos \theta \sin \theta \sin \theta_{u} \\
\sin \theta \cos \theta \cos \theta_{u}+\sin ^{2} \theta \sin \theta_{u}
\end{array}\right] \\
& =\|u\|\left(\cos \theta \cos \theta_{u}+\sin \theta \sin \theta_{u}\right)\left[\begin{array}{c}
\cos \theta \\
\sin \theta
\end{array}\right]
\end{aligned}
$$

This is just the familiar projection formula. Hence, the image is just the 1-dimensional subspace spanned by $\mathrm{e}^{i[\theta]}$.

The Kernel, substitution of $\theta_{u}$ by $\theta \pm \frac{\pi}{2}$ gives us the following result

$$
\begin{aligned}
& \|u\|\left(\cos \theta \cos \left(\theta+\frac{\pi}{2}\right)+\sin \theta \sin \left(\theta+\frac{\pi}{2}\right)\right)\left[\begin{array}{l}
\cos \theta \\
\sin \theta
\end{array}\right] \\
& =\|u\|(-\cos \theta \sin \theta+\sin \theta \cos \theta)\left[\begin{array}{l}
\cos \theta \\
\sin \theta
\end{array}\right]=0
\end{aligned}
$$

Hence, the kernel is the orthogonal complement of the subspace spanned by $\mathrm{e}^{i[\theta]}$. It is also easy to verify that $\mathrm{e}^{i[\theta]} \otimes\left(\mathrm{e}^{i[\theta]}\right)^{\mathrm{T}}$ is idempotent.

\section{Projections as a Topological Manifold}

Clearly, the topology $\left(\mathbb{S}^{1}, \cdot, \tau_{\mathbb{S}^{1}}\right)$ induces a topological structure on the projector group $G_{p}([\theta])$. 
Lemma

The topology $\left(G_{p}([\theta]), \tau_{G_{p}}\right)$ is Hausdorff and Second Countable.

Proof

Suppose we have 2 points $\mathrm{e}^{i[\theta]}$ and $\mathrm{e}^{i\left[\theta^{\prime}\right]}$, there exists $\mathcal{O}_{\mathbb{S}^{1}}^{\alpha}$ such that $\mathrm{e}^{i[\theta]} \in \mathcal{O}_{\mathbb{S}^{1}}^{\alpha}$ and an open arc $\mathcal{O}_{\mathbb{S}^{1}}^{\alpha^{\prime}}$ such that $\mathrm{e}^{i\left[\theta^{\prime}\right]} \in \mathcal{O}_{\mathbb{S}^{1}}^{\alpha}$ such that $\mathcal{O}_{\mathbb{S}^{1}}^{\alpha} \cap \mathcal{O}_{\mathbb{S}^{1}}^{\alpha^{\prime}}=\varnothing$. Hence, by the group homomorphism we

$$
\Phi\left(\mathcal{O}_{\mathbb{S}^{1}}^{\alpha} \cap \mathcal{O}_{\mathbb{S}^{1}}^{\alpha^{\prime}}\right)=\Phi\left(\mathcal{O}_{\mathbb{S}^{1}}^{\alpha}\right) \cap \Phi\left(\mathcal{O}_{\mathbb{S}^{1}}^{\alpha^{\prime}}\right)=\varnothing
$$

Therefore, it is Hausdorff.

For the second countability property, we proceed as follows.

Starting by using a countable basis in $\mathbb{R}$ of the form $\left(\theta, \theta^{\prime}\right)$ where $\theta, \theta^{\prime} \in \mathbb{Q}$. Since $\mathbb{Q}$ is countable then the set

$$
\mathcal{M}:=\left\{\left(\theta, \theta^{\prime}\right) \in \mathbb{R} \mid \theta, \theta^{\prime} \in \mathbb{Q}\right\}
$$

Is a countable basis on $\mathbb{R}$. This implies that the mapping

$$
\exp \{i \mathcal{M}\}
$$

induces a second countable basis on $\left(\mathbb{S}^{1}, \cdot, \tau_{\mathbb{S}^{1}}\right)$, which in turn, implies that $\Phi$ induces a second countable on $G_{P}([\theta])$.

Hence, we have a topological manifold.

Lemma 2

The topological manifold $G_{p}([\theta])$ is homeomorphic to $\mathbb{R}$.

Proof

We can define an atlas as follows

$$
\mathcal{A}:=\left\{\left(\mathcal{P}_{\alpha}, \phi\right) \mid \Phi\left(\mathcal{O}_{\mathbb{S}^{1}}^{\alpha}\right)=\mathcal{P}_{\alpha} \in \tau_{G_{P}}, \phi: G_{P} \rightarrow \mathbb{R}\right\}
$$

where

$$
\phi\left(\mathrm{e}^{i[\theta]} \otimes\left(\mathrm{e}^{i[\theta]}\right)^{\mathrm{T}}\right)=[\theta] \in \mathbb{R} / 2 \pi \mathbb{Z} \rightarrow \mathcal{B}([\theta], \epsilon)
$$

The mapping is bijective since

$$
\phi\left(\mathrm{e}^{i[\theta]} \otimes\left(\mathrm{e}^{i[\theta]}\right)^{\mathrm{T}}\right)=\phi\left(\mathrm{e}^{i\left[\theta^{\prime}\right]} \otimes\left(\mathrm{e}^{i\left[\theta^{\prime}\right]}\right)^{\mathrm{T}}\right)
$$

Implies that

$$
[\theta]=\left[\theta^{\prime}\right] \Leftrightarrow \theta^{\prime}=\theta \pm 2 \pi k, k \in \mathbb{Z}
$$

Or

$$
\theta^{\prime}=\theta \pm k \pi, k \in \mathbb{Z}
$$

Since we can get the following result

$$
\mathrm{e}^{i\left[\theta^{\prime}\right]} \otimes\left(\mathrm{e}^{i\left[\theta^{\prime}\right]}\right)^{\mathrm{T}}=\mathrm{e}^{i[\theta \pm \pi k]} \otimes\left(\mathrm{e}^{i[\theta \pm \pi k]}\right)^{\mathrm{T}}
$$

We get the following matrix

$$
\left[\begin{array}{cc}
\cos ^{2}(\theta \pm \pi k) & \cos (\theta \pm \pi k) \sin (\theta \pm \pi k) \\
\sin (\theta \pm \pi k) \cos (\theta \pm \pi k) & \sin ^{2}(\theta \pm \pi k)
\end{array}\right]
$$


Now it is easy to show that

$$
\cos (\theta \pm \pi k)= \pm \cos \theta, \sin (\theta \pm \pi k)= \pm \sin \theta
$$

Therefore, we have

$$
\begin{aligned}
& {\left[\begin{array}{cc}
\cos ^{2}(\theta \pm \pi k) & \cos (\theta \pm \pi k) \sin (\theta \pm \pi k) \\
\sin (\theta \pm \pi k) \cos (\theta \pm \pi k) & \sin ^{2}(\theta \pm \pi k)
\end{array}\right]} \\
& =\left[\begin{array}{cc}
\cos ^{2} \theta & \cos \theta \sin \theta \\
\sin \theta \cos \theta & \sin ^{2} \theta
\end{array}\right]
\end{aligned}
$$

However, since $\mathcal{O}_{\mathbb{S}^{1}}^{\alpha}$ is bounded such that $d\left(\mathrm{e}^{i \theta}, \mathrm{e}^{i \theta^{\prime}}\right)<\pi$ implies that $\phi$ is bijective on each $\mathcal{P}_{\alpha}, \forall \alpha \in I$. It is easy to see that $\phi$ is also continuous hence it defines a homeomorphism. For the transition functions, we arbitrarily choose some $\mathcal{P}_{\alpha}, \mathcal{P}_{\alpha}^{\prime} \in \tau_{G_{P}}$ such that $\mathcal{P}_{\alpha} \cap \mathcal{P}_{\alpha}^{\prime} \neq \varnothing$ then we have

$$
\phi \circ \phi^{-1}\left(\mathcal{P}_{\alpha} \cap \mathcal{P}_{\alpha}^{\prime}\right)=\mathcal{B}([\theta, \epsilon]) \cap \mathcal{B}\left(\left[\theta^{\prime}\right], \epsilon\right)
$$

Is open in $\mathbb{R} / 2 \pi \mathbb{Z}$.

Theorem 3. The topological manifold $G_{P}([\theta])$ is a Lie Group.

Proof

Let

$$
\mu: G_{P}([\theta]) \times G_{P}([\theta]) \rightarrow G_{P}([\theta])
$$

Such that

$$
\mu\left(P_{[\theta]}, P_{\left[\theta^{\prime}\right]}\right) \rightarrow P_{\left[\theta+\theta^{\prime}\right]} \in G_{P}([\theta])
$$

And

$$
l\left(P_{[\theta]}\right) \rightarrow\left(P_{[\theta]}\right)^{-1}=P_{[-\theta]}
$$

Since the group operation is addition of angles $\theta$ and $\theta^{\prime}$ implies that $\mu$ is both continuous and $C^{\infty}$. Same argument applies to the inverse mapping. It also clear that elements of these matrices are smooth transcendental functions of $\theta$ which are also $C^{\infty}$. Hence, we have a Lie Group.

\section{Group Action of $\mathbb{Z}$ on $G_{P}([\theta])$}

We now define the following group action of $\mathbb{Z}$ on the Lie Group $G_{P}([\theta])$ in the following way

$$
\xi: \mathbb{Z} \times G_{P}([\theta]) \rightarrow G_{P}([\theta])
$$

Such that

$$
\xi\left(z, P_{[\theta]}\right)=P_{[z \theta]}
$$

Lemma 4. Let $[\theta] \in \mathbb{T}$ be some point in the circle group for some fixed $\theta \in \mathbb{R}$. Then $[z \theta], z \in \mathbb{Z}$ is a subgroup of $\mathbb{T}$.

Proof

The action of $\mathbb{Z}$ on $\mathbb{T}$ given by $[z \theta], z \in \mathbb{Z}$ generates a subgroup since 


$$
[z \theta]+\left[z^{\prime} \theta\right]=\left[z \theta+z^{\prime} \theta\right]=\left[\left(z+z^{\prime}\right) \theta\right] \in \mathbb{T}
$$

With the additive identity and additive inverse in $\mathbb{Z}$, we have a subgroup of $\mathbb{T}$.

\section{Conclusion}

In conclusion, we have demonstrated the link between the circle group, the circle and the projection group. There is much to do to continue developing the theory. We wish to continue on this topic in subsequent articles.

\section{Acknowledgements}

I wish to extend my gratitude for the all, the very helpful advice from Prof. Nigel Atkins from Kingston University who was kind enough to take my numerous phone calls despite his busy teaching schedule.

\section{Conflicts of Interest}

The author declares no conflicts of interest regarding the publication of this paper.

\section{References}

[1] The Circle Group by Prof Girardi The Circle Group.

[2] Roman, S. (2008) Advanced Linear Algebra. 3rd Edition, Springer, Berlin. https://doi.org/10.1007/978-1-4757-2178-2

[3] Tu, L.W. (2011) An Introduction to Manifolds. 2nd Edition, Springer, Berlin. https://doi.org/10.1007/978-1-4419-7400-6

[4] Jacobson, N. (1951) Lectures in Abstract Algebra I, Basic Concepts. Springer, Berlin. https://doi.org/10.1007/978-1-4612-9872-4

[5] Jacobson, N. (1955) Lectures in Abstract Algebra II. The Mathematical Gazette, 39, 76-77. https://doi.org/10.2307/3611127 